\title{
Cure depth in photopolymerization: Experiments and theory
}

\author{
Jim H. Lee, Robert K. Prud'homme, and Ilhan A. Aksay \\ Department of Chemical Engineering and Princeton Materials Institute, Princeton University, \\ Princeton, New Jersey 08544-5263
}

(Received 21 August 2000; accepted 27 September 2001)

\begin{abstract}
The depth of photocuring for a model resin system was investigated as a function of photoinitiator concentration. Direct measurements of gel thickness were made from thin films of cross-linked multifunctional methacrylate monomer. The monomer, 2,2-bis\{4-[2-hydroxy-3-(methacryloxy)propoxy]phenyl\}propane, was polymerized in a solution of trichloroethylene with an ultraviolet laser light source at $325 \mathrm{~nm}$. The monomer solutions were photocured using varying levels of both photonic energy and photoinitiator concentration. An optimal photoinitiator concentration that maximized the gel cure depth was observed. Additionally, two regimes were shown to exist in which the shrinkage (upon solvent removal) was minimized or maximized. A model was developed to probe the physics of the system. Good agreement with experiment was obtained, and the model may be employed to predict both the existence and location of the optimal photoinitiator concentration and the corresponding cure depth. The study showed that photoinitiator plays a significant role in controlling the quality and performance of the formed gel network, with special regard to thickness of cured layers. This has potential application to fields as diverse as industrially cured coatings and dental fillings, and more generally, 3-dimensional rapid prototyping techniques.
\end{abstract}

\section{INTRODUCTION}

One method of producing highly cross-linked networks is through the photoinitiated polymerization of multifunctional monomers. The kinetics of these systems have been studied extensively in recent years. ${ }^{1,2}$ Several parameters are found to influence the final monomerto-polymer conversions of these systems, such as temperature, light intensity, monomer functionality and reactivity, and photoinitiator concentration. However, the roles of these exact same parameters on cure depth, as opposed to overall bond conversion, have not been fully examined.

Our interest in these relationships stems from our work in stereolithography, which is a rapid prototyping technique for making 3-dimensional polymeric and ceramic objects using laser-initiated photopolymerization in solutions or colloidal suspensions. ${ }^{3-5}$ In stereolithographic fabrication, a computer-aided design representation of a complex 3-dimensional object is computationally "sliced" into a sequence of 2-dimensional sections. The stereolithography apparatus (SLA) rasters a laser beam across the surface of a liquid pool of photocurable resin (Fig. 1). The laser beam polymerizes the surface of the resin, replicating the 2-dimensional section. An elevator platform submerges the cured layer deeper into the resin pool, and the next layer is polymerized. By subsequently building each new layer above the preceding layer, the designed 3-dimensional object is replicated as a real solid structure. The depth of cure determines the thickness of the sections that can be fabricated and therefore the number of layers required to fabricate an object.

It is the effect of photoinitiator concentration and light intensity on cure depth that interests us. In a related vein, the formation and curing of dental fillings face similar issues. Typical practice is to apply the dental paste in thin layers, laboriously curing each layer with a hand-held light source to reduce the amount of residual monomer leachable into the mouth. ${ }^{6,7}$ Some of the dental literature have suggested adding more photoinitiator to indirectly enhance the curing depth by driving the conversion-it would be desirable to understand the precise effect of doing so. ${ }^{8,9}$

In our examination of the cure depths in photopolymerization, we have observed that as the photoinitiator concentration is increased, the cure depth initially increases but then shows a reversal and starts to decrease after reaching a critical, or optimal, photoinitiator concentration. Thus, the cure depth is maximized for a particular photoinitiator concentration. While the relationship between the cure depth and light intensity is well-known and has been quantified, the same is not true of the photoinitiator dependence. ${ }^{10,11}$

The fact that the total monomer conversion increases throughout the reaction emphasizes the difference in following the photopolymerization with conversion or other 
similar measures as opposed to isolating the curing depth of the gel. Standard techniques for the examination of the rate and extent of polymerization in photocurable polymers, such as Fourier transform infrared spectroscopy (FTIR), are based upon measurement of the degree of double bond conversion, which is used as an indicator for the extent of reaction. ${ }^{12,13}$ Other techniques like photocalorimetry attempt similar evaluations, although the double bond conversion is obtained from enthalpy measurements rather than through FTIR. ${ }^{14,15}$ When photoinitiator concentration is varied, photocalorimetry and FTIR give valuable insight into the bulk extent of reaction as a function of photoinitiator concentration. However, they only indirectly track the cure depth. This is due to the fact that a thin film, coupled with the experimental setup, results in an evenly distributed illumination of the sample surface in the calorimeter tray. ${ }^{16}$ In such cases, the activation of initiator molecules is uniform, and thus, the concentration of free radical sites is homogenous throughout the film. This issue has not been addressed directly because in the thin film approximation, only the total conversion is considered.

In understanding the curing depth dependence on photoinitiator concentration, we first define the cure depth as the depth to which a 3-dimensional gel network is formed during photopolymerization. Coupled with this idea is the concept of a critical conversion for gelation. ${ }^{17-20}$ Since photon propagation through the medium is graded rather than discretized, gel is only formed up to the point at which the degree of cross-linking and

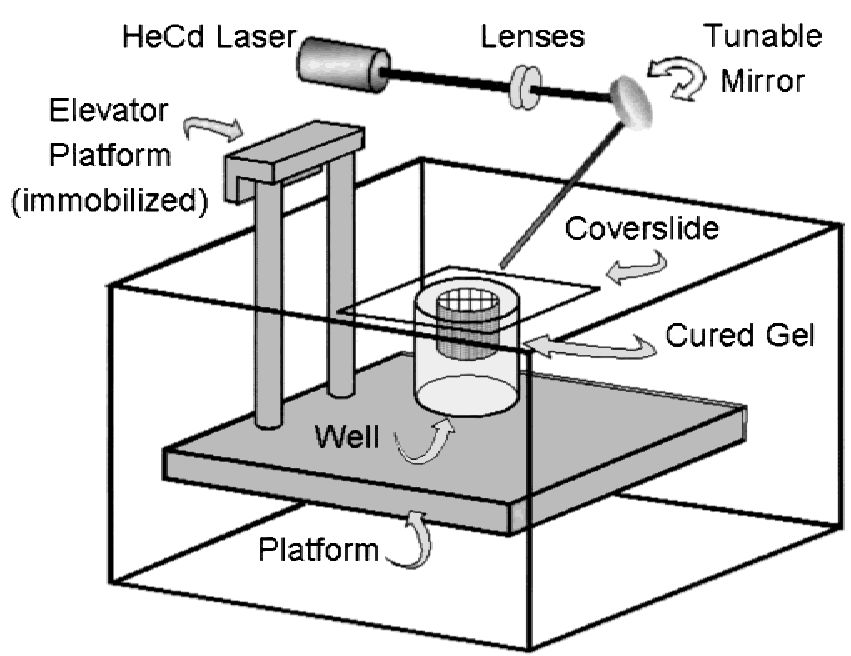

FIG. 1. Modified setup of the stereolithography apparatus. An articulated mirror directs the laser beam across the surface of the liquid monomer, creating a polymer gel network. Since our interest is in single layer films, we immobilize the elevator platform. A cylindrical well is placed on the platform, and the 60/40 weight ratio Bis-GMA/ trichloroethylene solution is pipetted inside. A glass coverslide on top acts as a substrate for gel attachment. The scanning velocity, and hence, energy dosage, is input via the stereolithography software. polymerization is sufficient to form a solid gel network. ${ }^{21,22}$ The curing depth thus corresponds to a thickness attainable via experimental measurement.

In this paper, we present a series of experiments that demonstrates the existence of a critical photoinitiator concentration for which the curing depth is maximized for photopolymerizations. We model this behavior by spatially coupling physical phenomena of light attenuation and critical conversions for gelation. The model qualitatively fits the experimentally observed dependence of cure depth on both light intensity and photoinitiator concentration. We show that the model developed not only supports the concept of a critical photoinitiator concentration for which the cure depth is maximized but allows for calculation of the optimal photoinitiator concentration and maximum curing depth attainable.

\section{EXPERIMENTAL PROCEDURE AND RESULTS}

The multifunctional monomer used in this study was 2,2-bis\{4-[2-hydroxy-3-(methacryloxy)propoxy]phenyl\}propane (Bis-GMA), a commonly employed monomer in the dental industry (Fig. 2). Bis-GMA was obtained from Polysciences, Inc., and was used as received. Photoinitiation was induced by an ultraviolet $(\mathrm{He}-\mathrm{Cd})$ laser at a wavelength of $325 \mathrm{~nm}$. The wavelength of the laser governs the choice of photoinitiator, and for these experiments, 2-benzyl-2-N,N-(dimethylamino)-1-(4morpholinophenyl)-1-butanone (DBMP) was employed (Fig. 2). DBMP has a molar extinction coefficient of approximately $23,000 \mathrm{M}^{-1} \mathrm{~cm}^{-1} \cdot{ }^{23}$ Photoinitiator (PI) concentration was varied from 0.34 to $99.70 \mathrm{mmol} / \mathrm{l}$ $(\mathrm{mM})$, corresponding to 0.01 to 3.00 weight percent (wt \%) of total solution and 0.02 to $5.00 \mathrm{wt} \%$ based on Bis-GMA monomer weight. These values are comparable to typical industrial formulations.

Trichloroethylene (TCE) was used as a solvent diluent in a 40/60 weight ratio with Bis-GMA by virtue of its being a strong solvent with minimal absorption at

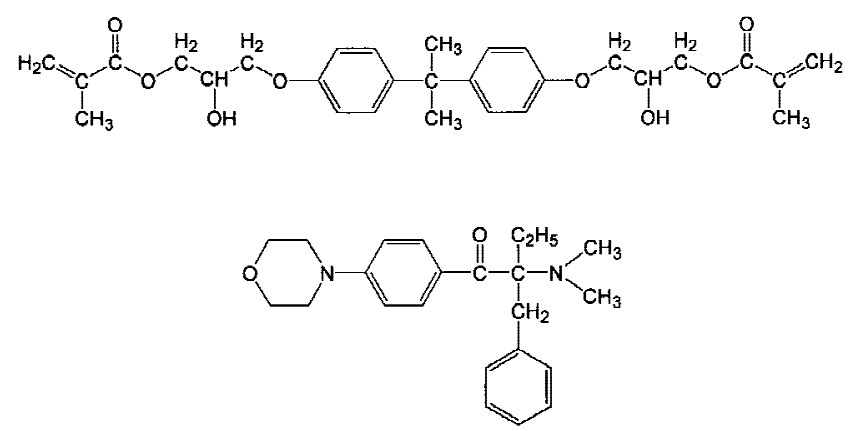

FIG. 2. Chemical structures for 2,2-bis\{4-[2-hydroxy-3(methacryloxy)propoxy]phenyl] propane (Bis-GMA) and 2-benzyl-2$N, N$-(dimethylamino)-1-(4-morpholinophenyl)-1-butanone (DBMP). Polymerization was initiated using light at a wavelength of $325 \mathrm{~nm}$. 
$325 \mathrm{~nm}\left(0.007 \mathrm{M}^{-1} \mathrm{~cm}^{-1}\right) .{ }^{24}$ The solution was allowed to mix in a sealed vial on a magnetic bar stir plate for a period of a few days with repeated washings of the wall with TCE to ensure homogenization. Excess solvent was removed by evaporation at room temperature to obtain a final 60/40 weight ratio of the monomer/solvent.

A 3D Systems Model 250 stereolithography apparatus was employed for the photopolymerizations. The SLA consists of a fixed wavelength lasing source with computer-controlled scanning velocity. The $40 \mathrm{~mW}$ ultraviolet $\mathrm{He}-\mathrm{Cd}$ laser has a Gaussian half-width of $125 \mu \mathrm{m} .{ }^{4,5}$ Our interest here is in single cured layers, so we fixed the elevator position and placed cylindrical wells on a platform (Fig. 1). The monomer/solvent solution was pipetted into the wells and filled up to the rim. A thin glass coverslide was placed on top, in contact with the solution. (The coverslides act as substrates for attachment during the polymerization and facilitate measurement of the gel thickness.)

Samples were cured by writing a cross-hatched pattern (Fig. 3) at three laser exposure levels of $0.931,1.702$, and $22.255 \mathrm{~J} / \mathrm{cm}^{2}$. The exposure levels were controlled by varying the laser beam writing speed via the stereolithography software. After polymerization, the glass coverslides were lifted off with the polymerized gel layer attached. The thicknesses of the cured gels were then measured $( \pm 0.01 \mathrm{~mm})$ with a micrometer in the "wet" state and then flushed with TCE to remove unreacted Bis-GMA monomer. After drying of the solvent overnight in a fume hood by evaporation at room temperature, the sample thicknesses were remeasured to obtain

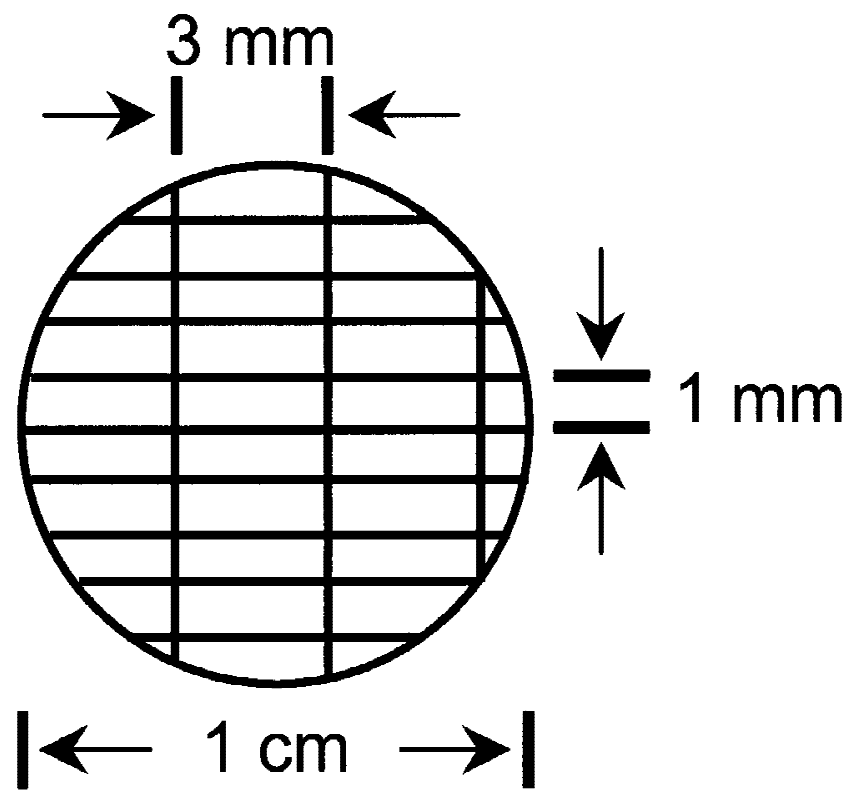

FIG. 3. Schematic of UV-cured disc. Black lines represent crosshatched rastering pattern of the $40 \mathrm{~mW}$ laser at 3 dosage levels: 0.931 , 1.702 , and $22.255 \mathrm{~J} / \mathrm{cm}^{2}$. The laser beam has a wavelength of $325 \mathrm{~nm}$ and a spot size of $250 \mu \mathrm{m}$. "dry" gel thicknesses. Residual solvent in the air-dried samples was no larger than $11 \%$, as measured by thermal gravimetric analysis. The "wet"-state cure depth is a measure of the depth of curing at conversions high enough to reach the gelation threshold, whereas the "dry"-state thickness is an indicator of the total polymerization conversion, or how much polymer is formed. This observed shrinkage thus provides information on the degree of cross-linking in the gel and holds implications for optimizing the stereolithography curing process, as discussed in Sec. IV. Two samples were produced for each concentration of photoinitiator for purposes of consistency.

The experimental results are shown in Figs. 4 and 5 for the "wet" and "dry" photopolymerized films. The ordinate is the gel thickness in millimeters, and the abscissa the photoinitiator concentration from 0.34 to $5.14 \mathrm{mM}$, which corresponds to 0.02 to $0.25 \mathrm{wt} \%$ based on BisGMA monomer weight. The corresponding wt $\%$ photoinitiator ( 0.01 to 0.15$)$ based on total weight of solution is shown on the top axis. The three data curves correspond to the three different laser energy dosages. Solid lines are calculated from the model developed in the next section for comparison at corresponding energy dosages. Continued "dark" gelation is expected to be minor due to rapid oxygen-scavenging of radicals. ${ }^{7,25}$ However, the effect is likely to be more pronounced at higher energy dosages, and thus the experimental deviation from theory is greater for the experimental data at $22.255 \mathrm{~J} / \mathrm{cm}^{2}$.

Extended data points were taken for higher photoinitiator concentrations to confirm that the cure depth decreases monotonically. In Fig. 6, the data range is extended to $0.15 \mathrm{M}$ (corresponding to $3 \mathrm{wt} \%$ solution and $5 \mathrm{wt} \%$ Bis-GMA) to more closely approach commercial formulations; $;{ }^{1,2}$ the cure depth continues to decrease and eventually plateaus. In the following section, a model is developed to probe the nonlinear dependence on photoinitiator concentration observed in Figs. 4 and 6.

\section{THEORETICAL MODEL FOR PHOTOCURING DEPTH}

We develop a model for photocuring depth by beginning with the kinetic equations for polymerization, ${ }^{26}$

$$
-\frac{\mathrm{d}[\mathrm{M}]}{\mathrm{d} t}=R_{\mathrm{i}}+R_{\mathrm{p}} \approx R_{\mathrm{p}}
$$

and

$$
R_{\mathrm{p}}=k_{\mathrm{p}}[\mathrm{M}]\left[\mathrm{M}^{*}\right],
$$

where $R_{\mathrm{p}}$ is the rate of polymerization, $R_{\mathrm{i}}$ is the rate of free radical initiation, $[\mathrm{M}]$ is monomer concentration, $\left[\mathrm{M}^{*}\right]$ is radical chain concentration, and $k_{\mathrm{p}}$ is the kinetic 
DBMP (weight \%, based on solution)

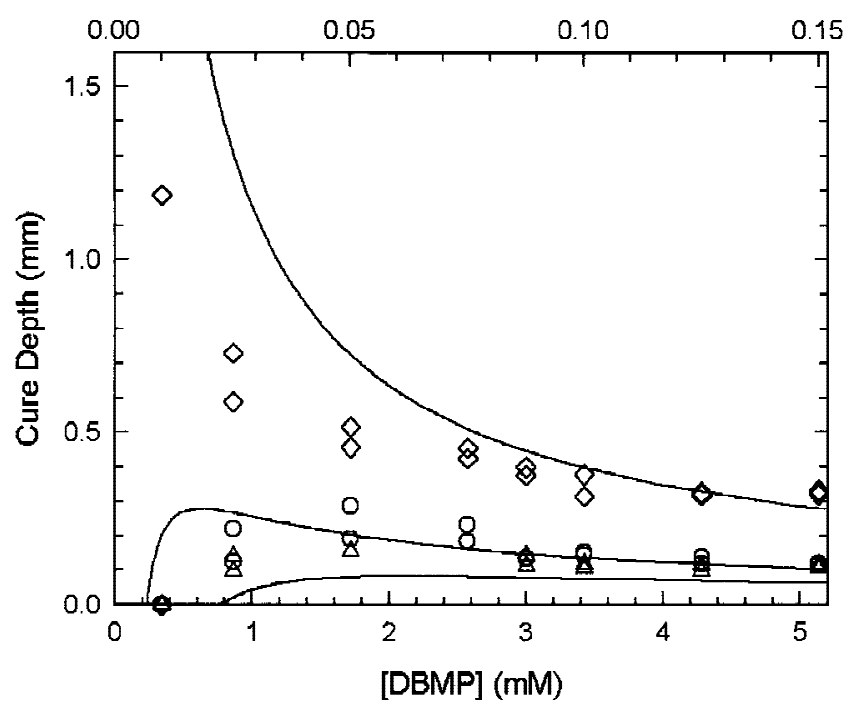

FIG. 4. Gel thickness versus photoinitiator concentration in the swollen "wet" state. The three data curves correspond to the three different laser energy dosages $\left(\diamond, 22.255 \mathrm{~J} / \mathrm{cm}^{2} ; \bigcirc, 1.702 \mathrm{~J} / \mathrm{cm}^{2} ; \triangle, 0.931\right.$ $\mathrm{J} / \mathrm{cm}^{2}$ ). Solid lines are calculated from the model for comparison at corresponding energy dosages $\left(\alpha=4.2 \times 10^{-6} \mathrm{M} \mathrm{s}^{0.5} \mathrm{~cm}^{0.5}\right)$. Photoinitiator concentration is given in $\mathrm{mmol} / \mathrm{l}(\mathrm{mM})$ on the bottom abscissa and as weight percent based on total solution on the top abscissa. Note that the model captures the concavity of the data and the location of the optimal photoinitiator concentration.

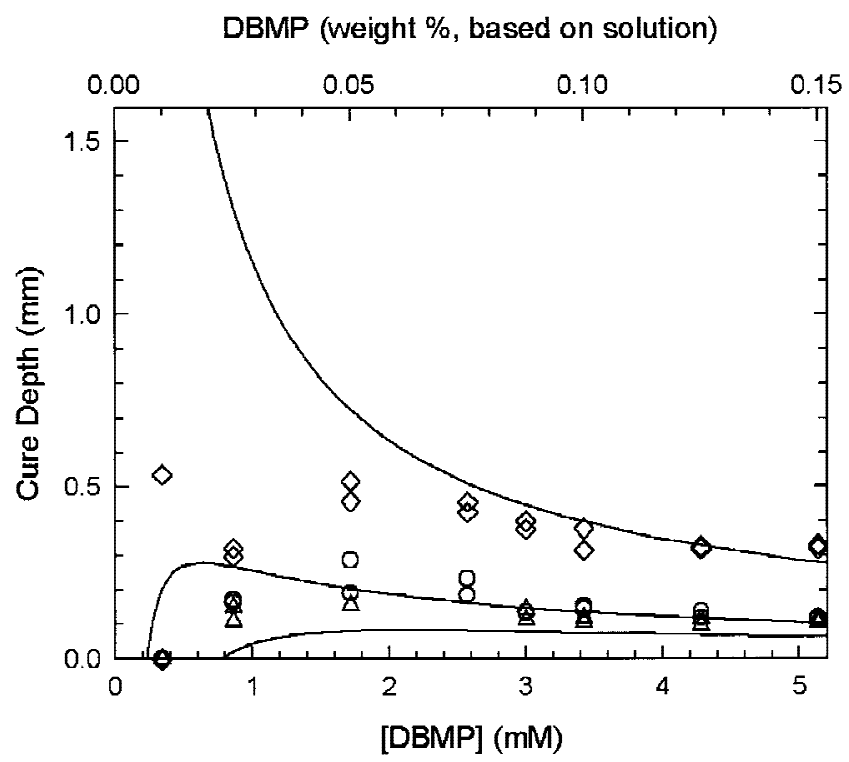

FIG. 5. Gel thickness versus photoinitiator concentration in the "dry" state. The three data curves correspond to the three different laser energy dosages $\left(\diamond, 22.255 \mathrm{~J} / \mathrm{cm}^{2} ; \bigcirc, 1.702 \mathrm{~J} / \mathrm{cm}^{2} ; \triangle, 0.931 \mathrm{~J} / \mathrm{cm}^{2}\right)$. Solid lines are calculated from the model for comparison at corresponding energy dosages $\left(\alpha=4.2 \times 10^{-6} \mathrm{M} \mathrm{s}^{0.5} \mathrm{~cm}^{0.5}\right)$. Photoinitiator concentration is given in $\mathrm{mmol} / \mathrm{l}(\mathrm{mM})$ on the bottom abscissa, and as weight percent based on total solution on the top abscissa. At low photoinitiator concentration, the gel network is loosely cross-linked and exhibits greater shrinkage upon drying than in the case of high photoinitiator concentration, where shrinkage is negligible.
DBMP (weight \%, based on solution)

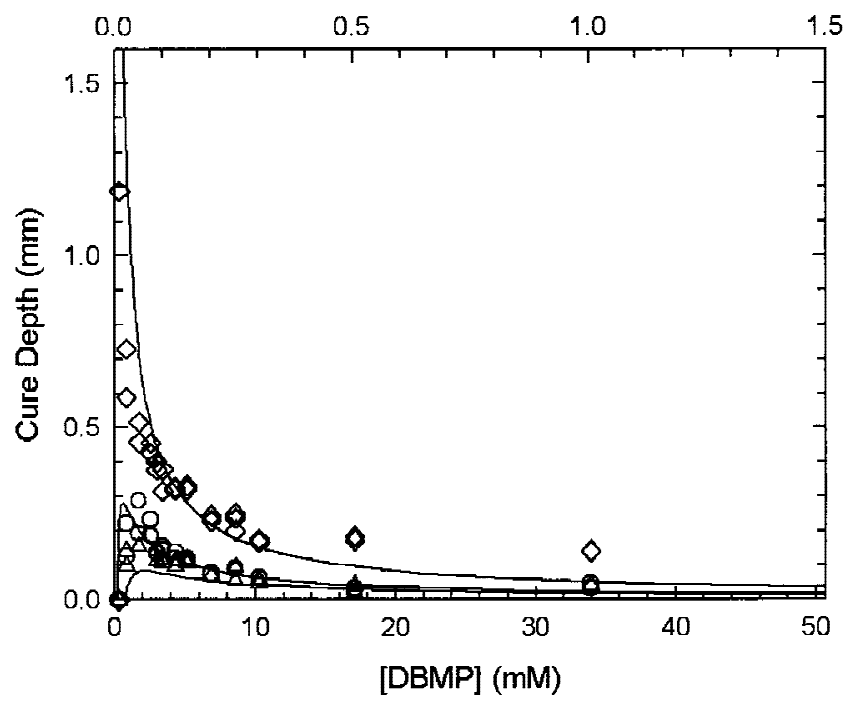

FIG. 6. Extended data for gel thickness versus photoinitiator concentration in the swollen "wet" state. The cure depth decreases monotonically as photoinitiator concentration increases without bound. The three data curves correspond to the three different laser energy dosages $\left(\diamond, 22.255 \mathrm{~J} / \mathrm{cm}^{2} ; \bigcirc, 1.702 \mathrm{~J} / \mathrm{cm}^{2} ; \triangle, 0.931 \mathrm{~J} / \mathrm{cm}^{2}\right)$. Solid lines are calculated from the model for comparison at corresponding energy dosages $\left(\alpha=4.2 \times 10^{-6} \mathrm{M} \mathrm{s}^{0.5} \mathrm{~cm}^{0.5}\right)$. Photoinitiator concentration is given in $\mathrm{mmol} / \mathrm{l}(\mathrm{mM})$ on the bottom abscissa and as weight percent based on total solution on the top abscissa.

rate constant for propagation. Employing the steady-state approximation (initiation of radicals equals termination), $R_{\mathrm{p}}$ is found as ${ }^{26}$

$$
R_{\mathrm{p}}=k_{\mathrm{p}}[\mathrm{M}]\left(\frac{R_{\mathrm{i}}}{2 k_{\mathrm{t}}}\right)^{1 / 2},
$$

where $k_{\mathrm{t}}$ is the kinetic rate constant for termination. For bifunctional photoinitiators, the initiation rate, $R_{\mathrm{i}}$ (Einsteins $1^{-3} \mathrm{t}^{-1}$ ), is related to the photonic flux, $I_{z}$ (Einsteins $\left.\mathrm{l}^{-2} \mathrm{t}^{-1}\right)$, by

$$
R_{\mathrm{i}}=2 \phi \epsilon[\mathrm{PI}] I_{z},
$$

where $\phi$ is the quantum yield of the photoinitiator, $\epsilon$ is the molar extinction coefficient $\left(\mathrm{M}^{-1} \mathrm{l}^{-1}\right)$, [PI] is the molar concentration of photoinitiator $(\mathrm{M})$, and $I_{z}$ is the incident photonic flux, or intensity, at depth $z$. The photonic flux at depth $z$ is related to the incident laser intensity at the surface $(z=0)$ by Beer's Law: ${ }^{27,28}$

$$
I_{z}=I_{0}\left(10^{-\epsilon[\mathrm{PI}] z}\right)
$$

and Eq. (4) may thus be written as

$$
R_{\mathrm{i}}=2 \phi \epsilon[\mathrm{PI}] I_{0}\left(10^{-\epsilon[\mathrm{PI}]_{z}}\right) .
$$

Substituting Eq. (6) into Eq. (3) allows the following expression to be obtained:

$$
-\frac{\mathrm{d}[\mathrm{M}]}{\mathrm{d} t} \approx R_{\mathrm{p}}=k_{\mathrm{p}}[\mathrm{M}]\left[\frac{\phi \in I_{0}[\mathrm{PI}]\left(10^{-\epsilon[\mathrm{PI}] z}\right)}{k_{\mathrm{t}}}\right]^{1 / 2} .
$$


Separating variables and integrating with the assumption of no time dependence in the bracketed term on the righthand side gives

$$
\ln \left(\frac{[\mathrm{M}]_{0}}{[\mathrm{M}]}\right)=\left[\frac{k_{\mathrm{p}}{ }^{2} \phi \in I_{0}[\mathrm{PI}]\left(10^{-\epsilon[\mathrm{PI}]_{z}}\right)}{k_{\mathrm{t}}}\right]^{1 / 2} t .
$$

The term in parentheses on the left-hand side is simply the degree of polymerization, ${ }^{17}$

$$
\bar{x}_{n}=\frac{[\mathrm{M}]_{0}}{[\mathrm{M}]} .
$$

The degree of polymerization is related to the extent of polymerization, $p$, by

$$
\bar{x}_{n}=\frac{1}{1-p}
$$

At the gel point, $p=p_{\mathrm{c}}$, the critical threshold for gelation. This threshold represents a critical conversion at which point a gel is formed. ${ }^{29,30}$ It therefore corresponds to the limit of the curing depth, $z_{\mathrm{c}}$, in the photocuring process and is a characteristic of the photochemical system. ${ }^{31,32}$ Substitution of Eqs. (9) and (10) into Eq. (8) at the gel point and rearrangement gives

$$
\left[\frac{k_{\mathrm{t}}}{k_{\mathrm{p}}^{2} \phi \in I_{0}}\right]\left[\ln \left(1-p_{\mathrm{c}}\right) / t\right]^{2}=[\mathrm{PI}]\left(10^{-\epsilon[\mathrm{PI}] z_{\mathrm{c}}}\right)
$$

Light intensity and exposure time are determined by the laser power and laser scanning speed, respectively. They are coupled to the maximum energy exposure per unit area, denoted $E_{\max }$ in the stereolithography literature, which is related (for a linearly translating Gaussian laser beam) to the power of the laser, $P_{\mathrm{L}}$, Gaussian halfwidth of the beam, $W_{0}$, and scanning velocity, $V_{\mathrm{S}}$, by ${ }^{4}$

$$
E_{\max }=\left(\frac{2}{\pi}\right)^{1 / 2} \frac{P_{\mathrm{L}}}{W_{0} V_{\mathrm{S}}}=\left(\frac{\operatorname{ch} N_{\mathrm{av}}}{\lambda}\right) I_{0} t
$$

where $c$ is the speed of light, $h$ is Planck's constant, $N_{\mathrm{av}}$ is Avogadro's number, and $\lambda$ is the wavelength of the laser. The exposure time is the time taken by the scanning laser beam to pass over a point on the surface: $t=$ $2 W_{0} / V_{\mathrm{S}}$. Substituting into Eq. (11) for $I_{0}$ and $t$, and eliminating $V_{\mathrm{S}}$ using Eq. (12), the following expression is obtained:

$$
\left[\frac{k_{\mathrm{t}}\left[\ln \left(1-p_{\mathrm{c}}\right)\right]^{2} \operatorname{ch} N_{\mathrm{av}} P_{\mathrm{L}}}{k_{\mathrm{p}}^{2} \phi \epsilon \lambda W_{0}^{2}(2 \pi)^{1 / 2}}\right]\left(\frac{1}{E_{\max }^{2}}\right)=[\mathrm{PI}]\left(10^{-\epsilon[\mathrm{PI}] z_{\mathrm{c}}}\right) .
$$

Recognizing that the term in the brackets on the left-hand side is comprised of constants of the chemical system and photopolymerization processing conditions, we collect terms and define variables $\alpha^{2}$ (that contains the photochemical parameters),

$$
\alpha^{2} \equiv \frac{k_{\mathrm{t}}\left[\ln \left(1-p_{\mathrm{c}}\right)\right]^{2}}{k_{\mathrm{p}}^{2} \phi \epsilon},
$$

and $\beta^{2}$ (which incorporates the stereolithography processing parameters).

$$
\beta^{2} \equiv \frac{\operatorname{ch} N_{\mathrm{av}} P_{\mathrm{L}}}{\lambda W_{0}^{2}(2 \pi)^{1 / 2}}
$$

Substituting Eqs. (14) and (15) into Eq. (13) allows the following expression to be obtained:

$$
\frac{\alpha^{2} \beta^{2}}{E_{\max }^{2}}=[\mathrm{PI}]\left(10^{-\epsilon[\mathrm{PI}] z_{\mathrm{c}}}\right)
$$

Solving Eq. (16) for $z_{\mathrm{c}}$,

$$
z_{\mathrm{c}}=\frac{2}{2.303 \epsilon[\mathrm{PI}]} \ln \left(\frac{E_{\max }[\mathrm{PI}]^{1 / 2}}{\alpha \beta}\right) .
$$

Jacobs has presented the "standard design equation" for stereolithography as

$$
C_{\mathrm{d}}=D_{\mathrm{p}} \ln \left(\frac{E_{\mathrm{max}}}{E_{\mathrm{c}}}\right),
$$

where $C_{\mathrm{d}}$ is the cure depth, $E_{\max }$ is the energy dosage per area, $E_{\mathrm{c}}$ represents a "critical" energy dosage, and $D_{\mathrm{p}}$ is the "depth of penetration" of the laser beam into the solution, which is inversely proportional to the molar extinction coefficient and concentration of photoinitiator. ${ }^{4,5}$ This empirical equation has been used in the literature $^{4,5}$ to analyze experimental data on cure depth versus laser writing speed to determine $D_{\mathrm{p}}$ and the empirical constant $E_{\mathrm{c}}$. These values are then used to determine the layer thickness of each layer for stereolithographic fabrication. Since the equation is not derived from photopolymerization chemistry, it suggests no dependence of $E_{\mathrm{c}}$ on photoinitiator concentration and does not predict a maximum in the cure depth curve with respect to photoinitiator concentration, which is the thrust of our contribution. Recognizing $z_{\mathrm{c}}$ as $C_{\mathrm{d}}$ and comparing Eqs. (17) and (18), we make the correspondence between the equations: the logarithmic dependence on $E_{\max }$ arises in both cases, and $D_{\mathrm{p}}$ is inversely proportional to $\epsilon$ and $[\mathrm{PI}]$ as

$$
D_{\mathrm{p}}\langle=\rangle \frac{2}{2.303 \epsilon[\mathrm{PI}]} \text {. }
$$


However, we find that the empirically derived "constant" term $E_{\mathrm{c}}$ is actually inversely dependent on the photoinitiator concentration to the one-half power, as written below:

$$
E_{\mathrm{c}}\langle=\rangle \frac{\alpha \beta}{[\mathrm{PI}]^{1 / 2}}
$$

Thus, it is $\alpha$ and $\beta$ that remain characteristic constants for a given photochemical system and processing conditions and not $E_{\mathrm{c}}$.

Interestingly, the model predicts a maximum in the cure depth versus photoinitiator concentration. Taking the derivative of $z_{\mathrm{c}}$ with respect to [PI],

$$
\begin{aligned}
\frac{\mathrm{d} z_{\mathrm{c}}}{\mathrm{d}[\mathrm{PI}]}= & \frac{1}{2.303 \epsilon[\mathrm{PI}]}\left(\frac{\alpha \beta}{E_{\max }[\mathrm{PI}]^{1 / 2}}\right) \frac{E_{\max }[\mathrm{PI}]^{-1 / 2}}{\alpha \beta} \\
& -\frac{2}{2.303 \epsilon[\mathrm{PI}]^{2}} \ln \left(\frac{E_{\max }[\mathrm{PI}]^{1 / 2}}{\alpha \beta}\right) .
\end{aligned}
$$

Setting to zero and solving for $[\mathrm{PI}]$ yields

$$
[\mathrm{PI}]_{\text {critical }}=\frac{2.718 \alpha^{2} \beta^{2}}{E_{\max }^{2}} .
$$

Note that as the energy dosage increases, the location of the maximum critical photoinitiator concentration nonlinearly shifts toward lower photoinitiator concentration. Furthermore, substitution of Eq. (22) into Eq. (17) yields that the depth of cure at the optimal photoinitiator concentration, $[\mathrm{PI}]_{\text {critical }}$, may be written as

$$
z_{\max }=\frac{E_{\max }^{2}}{6.259 \epsilon \alpha^{2} \beta^{2}} .
$$

Therefore, the theoretical model not only predicts the existence of a photoinitiator concentration that maximizes the depth of cure but predicts the maximum gel thickness as well.

A 3-dimensional map of the curing space as given by the model is shown in graphical form in Fig. 7. The gray mesh lines correspond to discretized photoinitiator concentration and energy dosage values, and for any given energy dosage, the figure exhibits a maximum cure depth at a specific photoinitiator concentration. Furthermore, as the energy dosage increases, the optimal photoinitiator concentration decreases, in accordance with Eq. (22). The magnitude of the maximum cure depth at a given energy dosage is obtained from Eq. (23). Note that only the region enclosed by the mesh lines represents physically realizable cure space. Mathematically, the black contour lines mark the boundary beyond which solutions of Eq. (17) incur complex values. Experimentally (as confirmed in the previous section and shown in Figs. 4-6), this corresponds to regions in which critical conversions are not obtained, and a coherent gel network is thus unable to form.

\section{COMPARISON BETWEEN EXPERIMENT AND THEORY}

The model above suggests the existence of a critical photoinitiator concentration for which the curing depth of the gel is maximized. Consideration of the 3-dimensional map of the curing topology thus sheds light on the shapes of the curing profiles at varying energy dosage levels shown in Figs. 4 and 6. As energy dosage varies, the location of the optimal photoinitiator concentration shifts to the left and skews the apex of the cure depth versus photoinitiator concentration curve.

We know all of the parameters that define $\beta^{2}$ in Eq. (15). We do not have values for the critical extent of polymerization $\left(p_{\mathrm{c}}\right)$ for gelation, the quantum yield $(\phi)$ for the photoinitiator at $325 \mathrm{~nm}$, and reaction rate constants $\left(k_{\mathrm{i}}\right.$ and $\left.k_{\mathrm{p}}\right)$ which comprise $\alpha^{2}$ in Eq. (14). Therefore we use $\alpha$ as a single fitting parameter to match experimental data. The nonlinear experimental dependence of cure depth on laser dosage and photoinitiator concentration is a relatively stringent test of the basic form of the model (Eq. 17), given that a single fitting parameter is used.

Often, the depth of cure is taken synonymously with the conversion, or extent of reaction. Literature shows an increase in monomer-to-polymer conversion with

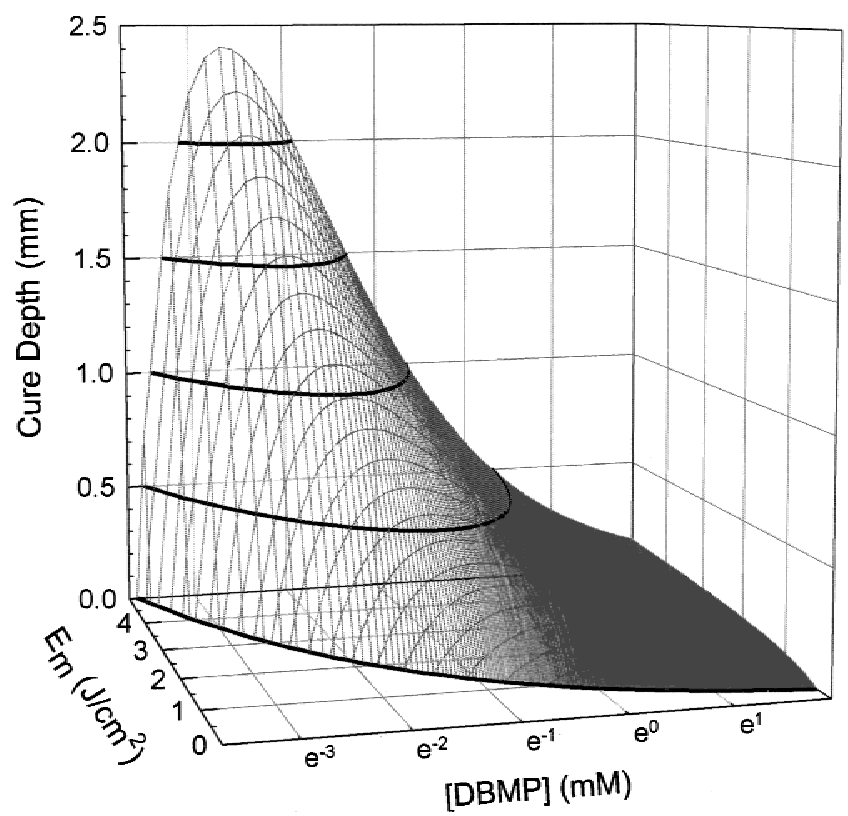

FIG. 7 Surface topology of the curing space. Photoinitiator concentration is plotted on the abscissa for emphasis, with energy dosage along the ordinate $\left(\alpha=4.2 \times 10^{-6} \mathrm{M} \mathrm{s}^{0.5} \mathrm{~cm}^{0.5}\right)$. Cure depth is shown upward in the $z$ direction. Note that cure depth increases exponentially as energy increases, while cure depth increases initially with increasing photoinitiator concentration, obtains a maximum, and then plateaus. The black contour lines mark the boundary of physically realizable cure space. 
increasing photoinitiator concentration; it is often implied that the cure depth follows conversion. ${ }^{8,13,16}$ Increasing the concentration of photoinitiator results in more radical initiation and, therefore, more double bond conversion. However, as photoinitiator concentration is raised, the penetration depth of the photons decreases. In the thin film approximation, the length scale of the medium is less than the penetration depth of light. When light attenuation is significant, however, the thin film assumption is invalid and the medium acts semiinfinitely; the penetration distance of the photons becomes less than the depth of the medium. Free radical initiation thus decreases with depth, and raising photoinitiator concentration results in localization of the free radical initiation closer to the surface. Since cure depth decreases with increasing photoinitiator concentration, at some point increasing photoinitiator concentration yields a smaller curing depth. At the other extreme, if the photoinitiator concentration is too low, then the conversion to polymer will be too low to form a gel. So the cure depth goes to zero as photoinitiator concentration approaches zero. Clearly, there must be an intermediate "optimal" concentration.

With reference to Figs. 4 and 5, another feature evident from the data is that the shrinkage upon drying decreases dramatically with increasing photoinitiator concentration. In the case of "low" photoinitiator concentration, the concentration of photoinitiator molecules is such that only a small fraction of the photons are absorbed. Thus, the penetration of light is greater than in the case of "high" photoinitiator concentration. Furthermore, because the concentration of photoinitiator molecules is low, the free radical concentration is subsequently lower as well. This results in a loosely crosslinked gel being formed, suggesting that, upon drying, the gel should shrink dramatically, as is the case shown in the data at low photoinitiator concentration as schematized in Fig. 8.

At "high" photoinitiator concentrations, photon absorption is greater, and the penetration depth of the laser is smaller. The free radical concentration is thus localized near the surface of the resin, which results in a tightly cross-linked, thin, cured profile. The data show that shrinkage in this regime decreases as the photoinitiator concentration increases. The degree of shrinkage (usually measured inversely as the swell ratio) is an important aspect of the curing process, because it affects the final dimensions of the cured sample. The minimum cure depth in stereolithography is defined by the finite step size of the elevator platform. Therefore, larger curing depths in the wet state facilitate lamination of layers and must be weighed against considerations of shrinkage in the final manufactured product. It is thus desired to minimize swell ratio and maximize cure depth.
Earlier literature examining the dependence of the curve depth on photoinitiator concentration was motivated by the idea of a crossover point or invariant curves for which existed a critical energy dosage and cure depth, denoted as $E^{*}$ and $C_{\mathrm{d}}^{*}{ }^{4,5,33,34}$ Equations may then be derived, given photoinitiator concentration for at least one known set of values for $D_{\mathrm{p}}$ and $E_{\mathrm{c}}$, for solutions of varying photoinitiator concentration. The derivations of these equations and their assumptions are shown in the Appendix, since they have not been reported elsewhere and are nontrivial. The equations (misprinted in Bernhard et al., 1993) are as follows:

$$
E^{*}=E_{\mathrm{c}_{1}}\left(\frac{[\mathrm{PI}]_{1}}{[\mathrm{PI}]_{2}}\right)^{\left[\frac{[\mathrm{PI}]_{1}}{[\mathrm{PI}]_{1}-[\mathrm{PI}]_{2}}\right]}
$$

and

$$
C_{\mathrm{d}}^{*}=D_{\mathrm{p}_{1}}\left(\frac{[\mathrm{PI}]_{1}}{[\mathrm{PI}]_{1}-[\mathrm{PI}]_{2}}\right) \ln \left[\frac{[\mathrm{PI}]_{1}}{[\mathrm{PI}]_{2}}\right],
$$

where the subscripts refer to two formulations of differing photoinitiator concentration. Special note should be taken that these equations do not in fact suggest that the photosensivity curves will be invariant as suggested in Jacobs. ${ }^{4,5}$ Nor do they result in a single, constant invariant crossover point as suggested in Bernhard et al. ${ }^{33,34}$ Rather, they simply represent the mathematical intersection of two lines with differing $D_{\mathrm{p}}$. The idea of a crossover point is misleading because this point shifts
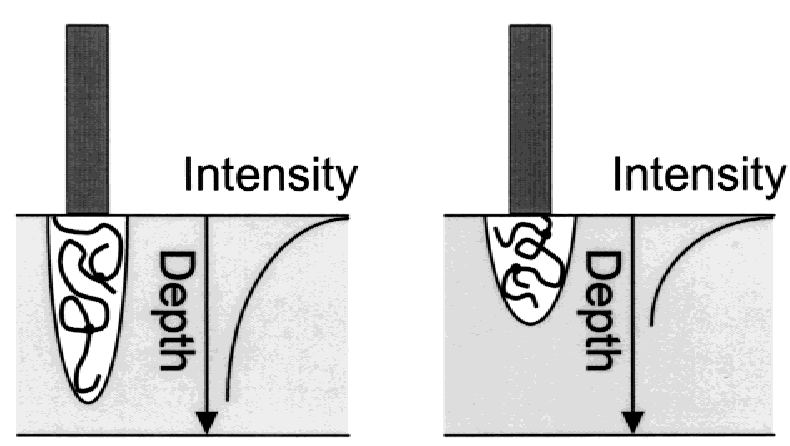

(a)
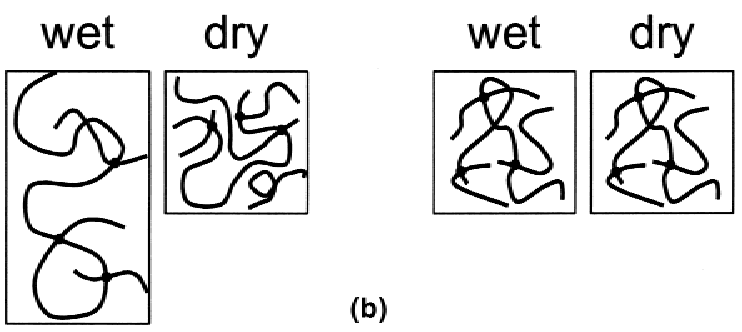

(b)

FIG. 8. Representation of gel curing profiles. In (a), laser penetrates deeply but only lightly cross-links the gel. In (b), free radical initiation is localized in upper portion of the solution, resulting in a thin but tightly cross-linked gel that undergoes little shrinkage upon drying. 
according to the two solutions being compared, and thus this model does not adequately account for the photoinitiator dependence.

In Figs. 4-6, the solid lines shown are calculated from the theoretical model for comparison with experimental results. Strong agreement is shown qualitatively, with the model accurately following the concavity of the curve for the three energy levels studied. In addition, the experimental data confirm that there exists a region where gelation does not occur, as evidenced by the continued formation of gel at high energy dosage levels, whereas, at low energy levels, the gel thickness drops to zero. For a given energy dosage, the model closely predicts both the location of the optimal photoinitiator concentration along the abscissa, as well as the magnitude of the cure depth. The model thus offers strong predictive capability for the effect of cure depth versus photoinitiator concentration.

\section{CONCLUSIONS}

In this work, the effect of photoinitiator concentration and laser dosage on cure depth of a polymerized gel network has been examined both experimentally and theoretically. The theoretical model clarifies the relationship between curing depth, energy dosage, and photoinitiator concentration for photopolymerization. The model quantifies and expands beyond qualitative explanations that have embedded the curing depth into measurements of overall double bond conversion as indicators of cure depth. The robustness of the model has been probed with experiments and shown to match curing thickness well.

Other published literature has suggested that the photoinitiator concentration may always be increased with the positive effect of increasing double bond conversion, with the implication that cure depth is increasing commensurately. ${ }^{8,9}$ In contrast, our model predicts the existence of a critical photoinitiator concentration that optimizes the curing depth for a given energy dosage. Our identification of optimal photoinitiator concentrations that maximized cure depth in our experiments supports this idea.

Since it is the final mechanical properties of the gel network that make it a useful material, it is also necessary to understand the quality of the gel network formed. The experimental results corroborate the expectation of the model that there should be two different regions of curing quality in which a cross-link density induces different properties in the swell ratio. Because gel shrinkage usually introduces residual stresses into the polymer, it is often desirable to minimize the swell ratio to produce a more homogeneous polymer gel network. These results provide a clear basis for optimizing the cure of these systems by controlling not only the depth of cure but minimizing shrinkage as well.
Our model for the photopolymerization includes the kinetic steps of initiation, propagation, and termination and also the critical degree of conversion to reach the gel point. The phenomena of oxygen quenching of radicals, polymerization autoacceleration, and detailed predictions of monomer functionality on the gel point have not been incorporated into the model. ${ }^{17-20,29-32,35,36}$ Future experiments to determine the remaining chemical kinetic parameters of $\alpha^{2}$ would allow an assessment of the significance of these other phenomena that have not been included and would serve as a great complement to the model.

\section{ACKNOWLEDGMENTS}

This work was funded by the Army Research Office under a MURI Grant No. DAAH04-95-1-0102. Partial support for the work was obtained from Johnson \& Johnson (CBC), the New Jersey Center for Biomaterials, and 3D Systems.

\section{REFERENCES}

1. J.P. Fouassier, Photoinitiation, Photopolymerization, and Photocuring (Hanser, Cincinnati, OH, 1995).

2. J.P. Fouassier and J.F. Rabek, Radiation Curing in Polymer Science and Technology (Elsevier, New York, 1993).

3. R. Garg, R.K. Prud'homme, I.A. Aksay, F. Liu, and R.R. Alfano, J. Mater. Res. 13, 3463 (1998).

4. P.F. Jacobs, Rapid Prototyping and Manufacturing (Society of Manufacturing Engineers, Dearborn, MI, 1992).

5. P.F. Jacobs, Stereolithography and other RP\&M Technologies (Society of Manufacturing Engineers, Dearborn, MI, 1996).

6. C.F. Cox, C.L. Keall, H.J. Keall, E. Ostro, and G. Bergenholtz, J. Prosthet. Dent. 57, 1-8 (1987).

7. K.S. Anseth, S.M. Newman, and C.N. Bowman, Adv. Polym. Sci. 122, 177 (1995).

8. F.A. Rueggeberg, P.E. Lockwood, and J.W. Ergle, J. Dent. Res. 76, 472 (1997).

9. F.A. Rueggeberg, J.W. Ergle, and P.E. Lockwood, Dent. Mater. 13, 360 (1997).

10. Y. Otsubo, T. Amari, and K. Watanabe, J. Appl. Polym. Sci. 29, 4071 (1984)

11. Y. Otsubo, T. Amari, and K. Watanabe, J. Appl. Polym. Sci. 31, 2099 (1986).

12. J.L. Ferracane and E.H. Greener, J. Dent. Res. 63, 1093 (1984).

13. K. Yoshida and E.H. Greener, J. Dent. 22, 296 (1994).

14. L. Lecamp, B. Youssef, C. Bunel, and P. Lebaudy, Polymer 38, 6089 (1997).

15. C. Hoyle, Radiation Curing: Science and Technology (Plenum Press, New York, 1992).

16. G.A. Brady and J.W. Halloran, J. Mater. Sci. 33, 4551 (1998).

17. P.J. Flory, Principles of Polymer Chemistry (Cornell University Press, Ithaca, NY, 1953).

18. M. Rubinstein and R.H. Colby, Macromolecules 27, 3184 (1994).

19. M. Gottlieb and C.W. Macosko, Macromolecules 15, 535 (1982). 
20. M. Muthukumar and H.H. Winter, Macromolecules 19, 1284 (1986).

21. J.S. Young, A.R. Kannurpatti, and C.N. Bowman, Macromol. Chem. Phys. 199, 1043 (1998).

22. A.R. Kannurpatti and C.N. Bowman, Macromolecules 31, 3311 (1998).

23. Ciba Specialty Chemicals, Photoinitiators for Curing: A Formulator's Guide (Ciba, Tarrytown, New York, 1997), p. 21.

24. Aldrich, Catalog Handbook of Fine Chemicals (Milwaukee, WI, 1998-99), p. 1639.

25. H.J. Hageman and L.G. Jansen, Makromol. Chem. 189, 2781 (1988).

26. G.G. Odian, Principles of Polymerization, 3rd ed. (Wiley, New York, 1991).

27. J.H. Lambert, Photometrie (Augsburg, Germany, 1760).

28. A. Beer, Ann. Physik Chem. 2, 78 (1852).

29. S.P. Obukhov, M. Rubinstein, and R.H. Colby, Macromolecules 27, 3191 (1994).

30. A. Hale, C.W. Macosko, and H.E. Bair, Macromolecules 24, 2610 (1991).

31. G.L. Batch and C.W. Macosko, Thermochim. Acta 166, 185 (1990).

32. J.E. Martin and D. Adolf, Annu. Rev. Phys. Chem. 42, 311 (1991).

33. P. Bernhard, M. Hofmann, M. Hunziker, B. Klingert, A. Schulthess, and B. Steinmann, in Radiation Curing in Polymer Science and Technology, edited by J.P. Fouassier and J.F. Rabek (Elsevier Applied Science, New York, 1993), Vol. IV, pp. 195-236.

34. P. Bernhard, M. Hofmann, A. Schulthess, and B. Steinmann, Chimia 48, 427 (1994).

35. G.A. O'Neil, M.B. Wisnudel, and J.M. Torkelson, Macromolecules 31, 4537 (1998).

36. G.A. O'Neil and J.M. Torkelson, Macromolecules 32, 411 (1999).

\section{APPENDIX: DERIVATION OF INVARIANT EQS. (24) AND (25)}

The equation for cure depth in stereolithography as written in Jacobs is ${ }^{4,5}$

$$
C_{\mathrm{d}}=D_{\mathrm{p}} \ln \left(\frac{E_{\mathrm{max}}}{E_{\mathrm{c}}}\right)
$$

For an invariant cure depth and energy dosage, we consider two formulations with differing photoinitiator concentrations. Denoting these two solutions with the subscripts 1 and 2, we may rewrite Eq. (A1) for each as

$$
C_{\mathrm{d}}=D_{\mathrm{p}_{1}} \ln \left(\frac{E_{\mathrm{max}}}{E_{\mathrm{c}_{1}}}\right), \quad C_{\mathrm{d}}=D_{\mathrm{p}_{2}} \ln \left(\frac{E_{\mathrm{max}}}{E_{\mathrm{c}_{2}}}\right) .
$$

Setting these equal and substituting for the depth of penetration,

$$
D_{\mathrm{p}}=\frac{\log \left(\frac{I_{0}}{I_{\mathrm{t}}}\right)}{\epsilon[\mathrm{PI}]},
$$

results in

$$
\frac{[\mathrm{PI}]_{2}}{[\mathrm{PI}]_{1}}=\frac{\ln \left(\frac{E_{\mathrm{max}}}{E_{\mathrm{c}_{2}}}\right)}{\ln \left(\frac{E_{\mathrm{max}}}{E_{\mathrm{c}_{1}}}\right)} .
$$

An assumption is made from a qualitative argument that ${ }^{4}$

$$
E_{\mathrm{c}_{1}}[\mathrm{PI}]_{1}=E_{\mathrm{c}_{2}}[\mathrm{PI}]_{2} \text {. }
$$

Then,

$$
\frac{[\mathrm{PI}]_{2}}{[\mathrm{PI}]_{1}}=\frac{\ln \left(\frac{E_{\mathrm{max}}}{E_{\mathrm{c}_{1}}} \frac{[\mathrm{PI}]_{2}}{[\mathrm{PI}]_{1}}\right)}{\ln \left(\frac{E_{\max }}{E_{\mathrm{c}_{1}}}\right)},
$$

and solving, Eq. (24) is obtained.

$$
E_{\max }=E_{\mathrm{c}_{1}}\left(\frac{[\mathrm{PI}]_{1}}{[\mathrm{PI}]_{2}}\right)\left[\frac{[\mathrm{PI}]_{1}}{[\mathrm{PI}]_{1}-[\mathrm{PI}]_{2}}\right] \text {. }
$$

If Eq. (A1) is recalled, the second invariant Eq. (25) is obtained through substitution. 